\title{
INFLUENCIA DA IDADE E DA POSIÇÃO RADIAL NAS DIMENSÕES DAS FIBRAS E DOS VASOS DA MADEIRA DE Eucalyptus grandis Hill ex. Maiden ${ }^{1}$
}

José de Castro Silva², Mário Tomazello Filho ${ }^{3}$, José Tarcísio da Silva Oliveira ${ }^{4}$ Vinícius Resende de Castro ${ }^{5}$

\begin{abstract}
RESUMO - Objetivou-se neste trabalho avaliar a variação das dimensões das fibras e dos vasos da madeira de Eucalyptus grandis com quatro diferentes idades (10, 14, 20 e 25 anos), proveniente de talhões comerciais. A amostragem do material na árvore foi feita através da coleta de três discos, retirados da base e das extremidades das duas primeiras toras, ambas com o comprimento comercial de $3 \mathrm{~m}$. De cada disco, retiraram-se cinco amostras, de dimensões $1,0 \times 1,0 \times 1,0 \mathrm{~cm}$, tomadas de pontos eqüidistantes, correspondentes a $0 ; 25 ; 50$; 75 ; e $100 \%$ da seção, no sentido radial medula-casca, fazendo-se a medição das fibras e dos vasos. Verificouse, em todos os parâmetros, o efeito da idade e da variação radial, no sentido medula-casca, à exceção da largura e do diâmetro do lume das fibras; todos os demais parâmetros apresentaram correlação positiva.
\end{abstract}

Palavras-chave: Eucalyptus grandis, anatomia e idade.

\section{INFLUENCE OF AGE AND RADIAL POSITION ON FIBER AND VESSEL DIMENSIONS OF Eucalyptus grandis Hill ex. Maiden WOOD}

\begin{abstract}
The objective of this work was to evaluate the variability of wood quantitative anatomical characteristics (fibers and vessels) of Eucalyptus grandis of four different ages (10, 14, 20 and 25 years), obtained from commercial stands. Tree material sampling was collected from three disks in the bottom and top of the first two logs, both three $m$ long. Five samples were collected from each disc at the pith-to-bark direction at 0 , 25, 50, 75 and 100\% of the radii. In all cases, age and pith-to-bark direction were observed to have a marked influence on all the assays, except for fiber lumen diameter and width; all the other parameters showed a positive correlation.
\end{abstract}

Keywords: Eucalyptus grandis, anatomy and age.

\section{INTRODUÇÃO}

Os estudos anatômicos possibilitam a identificação das espécies e, mais do que isso, informações sobre a estrutura do lenho, permitindo identificar as relações entre o lenho e as características gerais da madeira, principalmente nos aspectos referentes a resistência mecânica, permeabilidade, resistência natural e trabalhabilidade.
Segundo Marcati (1992), numerosos fatores, tanto internos quanto externos à árvore, conduzem a variações quanto ao tipo, número, tamanho, forma, estrutura física e composição química dos elementos. A sua estrutura é caracterizada pelo arranjo e quantidade proporcional de diferentes tipos de células, como fibras, traqueídeos, vasos, parênquima axial e raios, influenciando, significativamente, as diversas propriedades da madeira.

\footnotetext{
${ }^{1}$ Recebido em 25.04.2006 e aceito para publicação em 12.06.2007.

${ }^{2}$ Departamento de Engenharia Florestal da Universidade Federal de Viçosa (UFV). E-mail: <jcastro@ ufv.br>.

${ }^{3}$ Departamento de Ciências Florestais da Escola Superior de Agricultura Luiz de Queiroz(ESALQ/USP). E-mail: <mtomazel@esalq.usp.br>.

${ }^{4}$ Departamento de Engenharia Rural da Universidade Federal do Espírito Santo (UFES). E-mail: <jtsilva@ npd.ufes.br>.

${ }^{5}$ Engenharia Florestal da UFV. E-mail: <vresende@ gmail.com>.
} 
Zobel e Bujtenen (1989) afirmaram que as características morfológicas das fibras variam significativamente entre e dentro das árvores e podem ser controladas geneticamente, bem como apresentar alterações, em função de diferentes práticas silviculturais e da alteração da idade de corte.

Segundo Moreira (1999), o comprimento das fibras é diretamente influenciado pelas divisões longitudinaistangenciais no câmbio, como resultado da taxa de hormônios da árvore, que pode variar com a sazonalidade, condições ambientais, fatores genéticos e idade da árvore. Segundo Tomazelo Filho (1985a), Andrade (1987) e Esteban et al. (2003), as células cambiais passam a produzir, durante certo tempo, elementos com maiores dimensões até atingir a sua estabilização, em idades mais avançadas, ao longo do raio. Em razão disso, há aumento no comprimento das fibras no sentido medulacasca, com o aumento da idade. Assim, as fibras localizadas nas camadas de crescimento, mais próximas da medula e a dado nível do tronco, apresentam menores dimensões do que aquelas localizadas nas camadas finais de crescimento, mais próximas da casca. Hans e Burley (1972), estudando a madeira de diversas espécies de eucalipto, verificaram uma variação crescente no comprimento das fibras, no sentido medula-casca, até atingir um valor constante, a partir dos nove a 10 anos de idade. Tomazello Filho (1985b) afirmou que, regra geral, o modelo de variação no sentido radial das árvores de eucalipto, com idades de 1 a 25 anos, é caracterizado pelo aumento nas dimensões das fibras, nos primeiros anos, e, após esse período, atinge a estabilização, em torno do oitavo ao décimo anel de crescimento.

Segundo Shimoyama (1990) e Esteban et al. (2002), a largura das fibras está relacionada a fatores genéticos e ambientais, sendo influenciada pela idade cambial e tendendo a aumentar no sentido medula-casca. $\mathrm{O}$ diâmetro do lume, por sua vez, está relacionado com a largura e espessura da parede das fibras. Assim, quanto maiores forem os seus valores, mais espaços vazios serão encontrados na madeira, indicando menor massa específica.

TomazelloFilho(1985b), Shimoyama(1990) e Moreira (1999) afirmaram que a espessura da parede das fibras está relacionada a fatores genéticos e ambientais, bem como à idade da árvore, tendendo a aumentar no sentido medula-casca. Os vasos variam quanto à freqüência, diâmetro, forma e arranjo dentro da árvore. No sentido medula-casca, ocorre aumento no diâmetro dos elementos de vasos e redução na sua freqüência ao longo dos anéis sucessivos de crescimento, tendendo à estabilização na madeira adulta.

Este trabalho teve como objetivo avaliar a variabilidade das dimensões das fibras e vasos da madeira de Eucalyptus grandis, de quatro diferentes idades $(10,14,20$ e 25 anos) e de diferentes posições radiais, no sentido medula-casca.

\section{MATERIAL E MÉTODOS}

O material utilizado neste estudo foi obtido de plantios comerciais de Eucalyptus grandis Hill ex. Maiden, com idades de 10, 14, 20 e 25 anos, procedentes da Fazenda Monte Alegre, da KLABIN Fabricadora de Papel e Celulose S. A., localizada no Município de Telêmaco Borba, no Estado do Paraná. Utilizaram-se quatro árvores de cada idade, totalizando 16 árvores. Para a medição das fibras, tomaram-se três discos, retirados da base e do final da primeira e segunda toras, ambas com o comprimento comercial de $3 \mathrm{~m}$, procedimento considerado convencional nos testes de caracterização de madeira adulta para usos sólidos (serraria). De cada disco, retirou-se uma bagueta radial, definindo cinco posições eqüidistantes $(0,25,50,75$ e $100 \%)$, no sentido medula-casca. Não se avaliou a variabilidade longitudinal, ao longo do tronco; para isso, utilizou-se uma porção de material de cada um dos três discos, formando uma porção única, mas respeitando-se cada posição da seção radial. Posteriormente, foram retirados fragmentos (palitos finos) e obteve-se um macerado, com solução de peróxido de hidrogênio, segundo o método preconizado por Dadswell (1972). Mediram-se 20 fibras, individualmente, determinando-lhes o comprimento, largura e diâmetro do lume. A espessura da parede foi obtida pela relação da metade da diferença entre a largura e o diâmetro do lume. A face transversal do restante de cada subamostra, utilizada para a retirada dos fragmentos, foi devidamente aparada no micrótomo e polida, sendo utilizada para medir o diâmetro tangencial; foram tomados quatro pontos da seção transversal do lenho, através de um sistema de captura de imagens.

O desenho experimental em relação ao número de amostras foi esquematizado da seguinte maneira:

\section{Medição de fibras}

. Idade - (4 árvores x 4 idades x 20 medições $)=$ 320 amostras 
. Posição radial - (4 árvores x 5 posições radiais x 20 medições $)=400$ amostras

. Interação - (4 árvores x 1 idade x 1 posição x 20 medições $)=80$ amostras

\section{Diâmetro dos vasos} amostras

. Idade - (4 árvores x 4 idades x 4 medições ) $=64$

. Posição radial - (4 árvores x 5 posições radiais x 4 medições $)=80$ amostras

. Interação - (4 árvores x 1 idade x 1 posição x 4 medições $)=16$ amostras

O delineamento experimental foi o inteiramente casualizado, procedendo-se à análise estatística, através da análise de variância e do teste de médias (Tukey), considerando-se os efeitos da variação da idade e da posição radial no sentido medula-casca, bem como a interação entre os efeitos idade x posição radial no sentido medula-casca. O coeficiente de variação experimental foi determinado através da fórmula CV

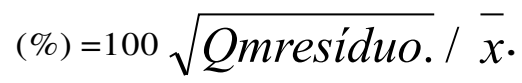

A Figura 1 apresenta o esquema de retirada das amostras para os ensaios de anatomia.

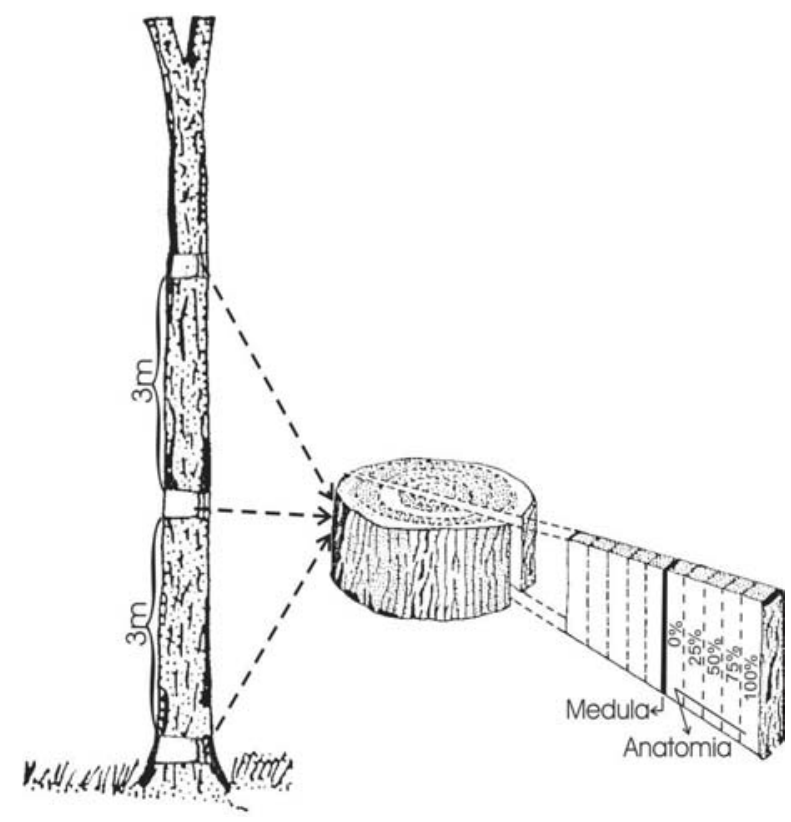

Figura 1 - Retirada das amostras para ensaios de anatomia. Figure 1 - Sample collection for anatomy assays.

\section{RESULTADOS E DISCUSSÕES}

\subsection{Comprimento das fibras}

Pela análise de variância, verificou-se que os efeitos da idade e, principalmente, da variação radial no sentido medula-casca foram significativos, em nível de 5\%, pelo teste F; a interação desses efeitos teve menor influência na definição dos valores, conforme os dados apresentados no Quadro 1.

As dimensões de comprimento das fibras estão apresentadas no Quadro 2.

Os valores encontrados no comprimento das fibras estão em conformidade com os normalmente encontrados na literatura, dentro da faixa estabelecida que varia de 0,75 a 1,40 mm. O valor médio do comprimento das fibras foi de $1,12 \mathrm{~mm}$, sendo os limites superior e inferior, respectivamente, de 1,43 $\mathrm{mm}$ (idade de 20 anos na posição mais próxima da casca) e $0,76 \mathrm{~mm}$ (idade de 10 anos na posição mais próxima da medula), com uma variação de $88,16 \%$. Os dados apresentados no Quadro 2 evidenciam um aumento no comprimento das fibras em função da variação da idade e, principalmente, do sentido radial medula-casca. Estatisticamente, observaram-se diferenças significativas entre os valores médios de comprimento de fibras para todas as idades e todas as posições. Verificou-se a maior variação dos valores $(8,82 \%)$ entre as madeiras de dez e quatorze anos, seguida da variação de $3,60 \%$ entre as madeiras de quatorze e vinte e cinco anos; entre as madeiras de vinte e vinte e cinco anos a variação foi de $3,47 \%$. Em relação à posição radial, verificou-se a maior variação dos valores entre as posições de 0 e $25 \%$, correspondente à região próxima da medula. Independentemente das diferentes idades, todos os valores médios encontrados nas posições mais próximas da medula foram inferiores à média geral.

\subsection{Largura das fibras}

A análise de variância da largura das fibras está apresentada no Quadro 3.

Pela análise de variância, verificou-se a influência da idade e, principalmente, da posição radial na largura das fibras, apresentando valores de F significativos; a influência da interação idade x posição radial também foi verificada, embora em menor intensidade.

R. Árvore, Viçosa-MG, v.31, n.6, p.1081-1090, 2007 
Quadro 1 - Análise de variância do comprimento das fibras da madeira de Eucalyptus grandis de diferentes idades e posições, no sentido radial medula-casca

Table 1 - Variance analysis of Eucalyptus grandis wood fiber length at different ages and distances in the bark-radial direction

\begin{tabular}{lcccc}
\hline Causa da Variação & G. L. & Soma quadrados & Q. médio & F \\
\hline A: Idade & 3 & 21,466 & 7,15532 & $230,07^{*}$ \\
B: Medula-casca & 4 & 107,79 & 26,9475 & $866,78^{*}$ \\
AB & 12 & 6,26747 & 0,522289 & $16,79 *$ \\
Resíduo & 4778 & 148,596 & 0,0311 & 0,0000 \\
\hline Total (Corrigido) & 4797 & 284,079 & & \\
\hline
\end{tabular}

C.V. experimental: $15,74 \%$.

Nota: * significativo a $95 \%$ de probabilidade.

Quadro 2 - Dimensões de comprimento de fibras ( $\mathrm{mm}$ ) da madeira de Eucalyptus grandis Hill ex. Maiden com diferentes idades (anos) e posições no sentido radial medula-casca

Table 2 - Eucalyptus grandis Hill ex. Maiden wood fiber length dimensions ( $\mathrm{mm}$ ) at different ages (years) and differences in the pith-to-bark direction

\begin{tabular}{|c|c|c|c|c|c|}
\hline Tratamento & $\mathrm{N}$ (amostras) & Médias & Tukey & $\mathrm{CV}(\%)$ & $\mathrm{DP}(\mathrm{mm})$ \\
\hline Idade 10 anos & 320 & 1,02 & $\mathrm{a}^{\mathrm{1}^{\prime \prime}}$ & 20,66 & 0,210 \\
\hline Idade 14 anos & 320 & 1,11 & $\mathrm{~b}$ & 20,33 & 0,226 \\
\hline Idade 25 anos & 320 & 1,15 & $\mathrm{c}$ & 19,94 & 0,230 \\
\hline Idade 20 anos & 320 & 1,19 & d & 22,18 & 0,226 \\
\hline Posição 0\% (M-C) & 400 & 0,87 & a & 19,51 & 0,169 \\
\hline Posição 25\% (M-C) & 400 & 1,04 & $\mathrm{~b}$ & 17,48 & 0,182 \\
\hline Posição 50\% (M-C) & 400 & 1,18 & $\mathrm{c}$ & 16,76 & 0,197 \\
\hline Posição 75\% (M-C) & 400 & 1,24 & $\mathrm{~d}$ & 16,32 & 0,202 \\
\hline Posição 100\% (M-C) & 400 & 1,28 & $\mathrm{e}$ & 16,06 & 0,206 \\
\hline \multicolumn{6}{|c|}{ Interação idade $\mathrm{X}$ posição radial medula-casca } \\
\hline 10 anos $x$ posição $0 \%$ & 80 & 0,78 & $\mathrm{a}$ & 19,01 & 0,149 \\
\hline 25 anos x posição $0 \%$ & 80 & 0,88 & $\mathrm{~b}$ & 17,24 & 0,153 \\
\hline 20 anos $x$ posição $0 \%$ & 80 & 0,89 & $\mathrm{~b}$ & 16,37 & 0,145 \\
\hline 14 anos x posição $0 \%$ & 80 & 0,92 & $\mathrm{~b}$ & 21,22 & 0,195 \\
\hline 10 anos x posição $25 \%$ & 80 & 0,98 & $\mathrm{c}$ & 14,84 & 0,146 \\
\hline 14 anos $x$ posição $25 \%$ & 80 & 1,05 & $\mathrm{~d}$ & 20,93 & 0,222 \\
\hline 10 anos $x$ posição $50 \%$ & 80 & 1,05 & d & 15,85 & 0,167 \\
\hline 20 anos x posição $25 \%$ & 80 & 1,06 & $\mathrm{~d}$ & 16,98 & 0,179 \\
\hline 25 anos x posição $25 \%$ & 80 & 1,08 & de & 14,88 & 0,161 \\
\hline 10 anos x posição $75 \%$ & 80 & 1,13 & ef & 15,55 & 0,175 \\
\hline 14 anos $x$ posição $50 \%$ & 80 & 1,15 & $f g$ & 17,43 & 0,201 \\
\hline 10 anos $x$ posição $100 \%$ & 80 & 1,15 & $\mathrm{fg}$ & 15,97 & 0,183 \\
\hline 14 anos x posição $75 \%$ & 80 & 1,19 & gh & 14,79 & 0,175 \\
\hline 25 anos x posição $50 \%$ & 80 & 1,21 & hi & 13,81 & 0,167 \\
\hline 14 anos x posição $100 \%$ & 80 & 1,24 & hij & 14,77 & 0,183 \\
\hline 25 anos $x$ posição $75 \%$ & 80 & 1,27 & $\mathrm{ij}$ & 14,65 & 0,186 \\
\hline 20 anos $x$ posição $50 \%$ & 80 & 1,28 & $\mathrm{j} 1$ & 13,93 & 0,178 \\
\hline 25 anos $x$ posição $100 \%$ & 80 & 1,32 & $\operatorname{lm}$ & 12,98 & 0,172 \\
\hline 20 anos x posição $75 \%$ & 80 & 1,37 & $\mathrm{mn}$ & 13,48 & 0,185 \\
\hline 20 anos x posição $100 \%$ & 80 & 1,41 & $\mathrm{n}$ & 13,14 & 0,185 \\
\hline
\end{tabular}

1/ Médias seguidas da mesma letra não diferem estatisticamente entre si, pelo teste de Tukey com significância de $5 \%$ ( $<<0,05$ ). $\mathrm{M}-\mathrm{C}=$ medula-casca; $\mathrm{CV}=$ coeficiente de variação $(\%)$; e DP = desvio-padrão.

R. Árvore, Viçosa-MG, v.31, n.6, p.1081-1090, 2007 
Quadro 3 - Análise de variância da largura das fibras da madeira de Eucalyptus grandis de diferentes idades e posições, no sentido radial medula-casca

Table 3 - Variance analysis of Eucalyptus grandis wood fiber width at different ages and distances in the pith-to-bark direction

\begin{tabular}{lccccc}
\hline Causa da variação & G. L. & Soma quadrados & Q. médio & F & P \\
\hline A: Idade & 3 & 2657,24 & 885,746 & $72,78^{*}$ & 0,0000 \\
B: Medula-casca & 4 & 4427,96 & 1106,99 & $90,96^{*}$ & 0,0000 \\
AB & 12 & 1012,75 & 84,3958 & $6,93^{*}$ & 0,0000 \\
Resíduo & 4778 & 58146,6 & 12,1697 & & \\
\hline Total (Corrigido) & 4797 & 66232,9 & & & \\
\hline
\end{tabular}

C.V. experimental: $17,65 \%$.

NOTA: * significativo a $95 \%$ de probabilidade.

Os valores de largura das fibras estão em conformidade com os normalmente encontrados na literatura, dentro da faixa estabelecida, que varia de 12 e $20 \mu \mathrm{m}$. O valor médio da largura das fibras foi de $19,77 \mu \mathrm{m}$, sendo os limites superior e inferior, respectivamente, de $22,74 \mu \mathrm{m}$ (idade de 10 anos, na posição mais próxima da casca) e 17,32 $\mu \mathrm{m}$ (idade de 20 anos, na posição radial mais próxima da medula), com uma variação de $31,29 \%$. Estatisticamente, não houve diferenças significativas entre os valores médios de largura de fibras nas idades de 10 e 14 anos; no entanto, elas diferiram nas idades de 20 e 25 anos.

Os dados do Quadro 4 apresentam diminuição na largura das fibras em função da idade, mas, em contrapartida, exibem um aumento das suas dimensões na direção radial.

Quadro 4 - Dimensões da largura das fibras (mm) da madeira de Eucalyptus grandis Hill ex. Maiden, de diferentes idades (anos) e posições radiais no sentido medula-casca

Table 4 - Fiber width dimensions ( $\mathrm{mm}$ ) of Eucalyptus grandis Hill ex. Maiden wood of different ages (years) and different distances in the pith-to-bark radial direction

\begin{tabular}{|c|c|c|c|c|c|}
\hline Tratamento & $\mathrm{N}$ (amostras) & Médias (largura de fibras) & Tukey & $\mathrm{CV}(\%)$ & $\mathrm{DP}(\mu \mathrm{m})$ \\
\hline Idade 20 anos & 320 & 18,74 & $\mathrm{a}^{\underline{1 /}}$ & 16,31 & 3,056 \\
\hline Idade 25 anos & 320 & 19,39 & $\mathrm{~b}$ & 16,90 & 3,278 \\
\hline Idade 10 anos & 320 & 20,41 & $\mathrm{c}$ & 20,20 & 4,121 \\
\hline Idade 14 anos & 320 & 20,55 & c & 19,46 & 3,998 \\
\hline Posição 0\% (M-C) & 400 & 18,11 & $\mathrm{a}$ & 16,39 & 2,969 \\
\hline Posição 25\% (M-C) & 400 & 19,32 & b & 18,64 & 3,600 \\
\hline Posição $50 \%$ (M-C) & 400 & 20,33 & $\mathrm{c}$ & 18,09 & 3,679 \\
\hline Posição 75\% (M-C) & 400 & 20,35 & $\mathrm{~d}$ & 17,93 & 3,648 \\
\hline Posição $100 \%$ (M-C) & 400 & 20,76 & $\mathrm{e}$ & 19,20 & 3,987 \\
\hline \multicolumn{6}{|c|}{ Interação idade X posição radial (medula - casca) } \\
\hline 25 anos x posição $0 \%$ & 80 & 17,76 & $\mathrm{a}$ & 15,16 & 2,692 \\
\hline 20 anos $x$ posição $0 \%$ & 80 & 17,77 & a & 14,50 & 2,584 \\
\hline 10 anos $x$ posição $0 \%$ & 80 & 17,82 & $\mathrm{a}$ & 15,56 & 2,775 \\
\hline 20 anos $x$ posição $25 \%$ & 80 & 17,83 & $\mathrm{a}$ & 15,83 & 2,612 \\
\hline 14 anos $x$ posição $0 \%$ & 80 & 19,03 & $\mathrm{~b}$ & 18,61 & 3,542 \\
\hline 20 anos $x$ posição $50 \%$ & 80 & 19,11 & $\mathrm{~b}$ & 15,03 & 2,872 \\
\hline 25 anos $x$ posição $25 \%$ & 80 & 19,43 & bc & 15,73 & 3,057 \\
\hline 20 anos $x$ posição $75 \%$ & 80 & 19,45 & bc & 16,23 & 3,174 \\
\hline 20 anos $x$ posição $100 \%$ & 80 & 19,56 & bc & 16,91 & 3,290 \\
\hline 25 anos $x$ posição $50 \%$ & 80 & 19,59 & $\mathrm{bc}$ & 15,08 & 2,986 \\
\hline 10 anos $x$ posição $25 \%$ & 80 & 19,81 & bcd & 20,393 & 4,040 \\
\hline 25 anos $x$ posição $75 \%$ & 80 & 19,81 & bcd & 17,84 & 3,637 \\
\hline 14 anos $x$ posição $25 \%$ & 80 & 20,25 & cde & 19,05 & 3,858 \\
\hline 25 anos $x$ posição $100 \%$ & 80 & 20,38 & cde & 17,15 & 3,360 \\
\hline 14 anos x posição $50 \%$ & 80 & 20,51 & cde & 19,40 & 4,123 \\
\hline 10 anos x posição $50 \%$ & 80 & 20,93 & def & 19,38 & 4,102 \\
\hline 10 anos x posição $75 \%$ & 80 & 21,17 & efg & 17,11 & 3,581 \\
\hline 14 anos $x$ posição $75 \%$ & 80 & 21,25 & efg & 19,66 & 4,034 \\
\hline 14 anos $x$ posição $100 \%$ & 80 & 21,71 & $\mathrm{fg}$ & 17,97 & 3,902 \\
\hline 10 anos $x$ posição $100 \%$ & 80 & 22,29 & $\mathrm{~g}$ & 20,11 & 4,483 \\
\hline Média Geral & & & 19,77 & & \\
\hline
\end{tabular}

1/ Médias seguidas da mesma letra não diferem estatisticamente entre si, pelo teste de Tukey com significância de $5 \%$ (p<0,05). ).

$\mathrm{M}-\mathrm{C}=$ medula-casca; $\mathrm{CV}=$ coeficiente de variação $(\%)$; e DP = desvio-padrão. 


\subsection{Diâmetro do lume}

Pela análise de variância, verificou-se a influência da posição radial e, principalmente, da idade no diâmetro do lume, apresentando valores de F significativos; com menor intensidade, tambév se verificou que o diâmetro do lume teve influência dos efeitos da interação entre idade e posição radial no sentido medula-casca, conforme o Quadro 5.

Observou-se diminuição no diâmetro do lume com o aumento da idade; no entanto, situação inversa foi observada no sentido medula-casca. Os valores encontrados para o diâmetro do lume das fibras foram ligeiramente superiores aos encontrados na literatura, que, segundo Silva (2002), variam de 6 a $12 \mu \mathrm{m}$. O valor médio do diâmetro do lume das fibras foi de $12,25 \mu \mathrm{m}$, sendo os limites individuais superior e inferior, respectivamente, de 15,06 $\mu \mathrm{m}$ (idade de 10 anos na posição mais próxima da casca) e 9,02 $\mu \mathrm{m}$ (idade de 25 anos na posição mais próxima da medula), com uma variação de $66,96 \%$, estatisticamente, mas não se observaram diferenças entre as idades de 10 e 14 anos; no entanto, elas diferiram entre a idade de 20 e 25 anos. Não se observaram diferenças significativas entre os valores médios das posições 0,25 e $50 \%$, mas tais diferenças foram verificadas entre as posições 50, 75 e $100 \%$, conforme o Quadro 6.

\subsection{Espessura da parede}

Pela análise de variância, verificaram-se efeitos significativos da idade e, principalmente, da variação radial no sentido medula-casca, na espessura da parede; a influência da interação dos efeitos idade $\mathrm{x}$ posição radial, no sentido medula-casca, também foi verificada.

Os dados do Quadro 8 indicam um aumento na espessura da parede em função da variação da idade e, principalmente, do sentido medula-casca. Os valores encontrados neste trabalho estão em conformidade com os normalmente encontrados na literatura, que variam de 2,5 a $6,0 \mu \mathrm{m}$. O valor médio da espessura da parede das fibras foi de $3,76 \mu \mathrm{m}$, sendo os limites individuais superior e inferior, respectivamente, de $5,21 \mu \mathrm{m}$ (idade de 20 anos na posição mais próxima da casca) e 2,88 $\mu \mathrm{m}$ (idade de 10 anos na posição mais próxima da medula), com uma variação de $80,9 \%$. De modo geral, observaram-se diferenças significativas entre todas as posições e entre todas as idades.

\subsection{Diâmetro tangencial dos vasos}

Pela análise de variância, verificaram-se efeitos significativos da idade e, principalmente, da variação radial no sentido medula-casca no diâmetro tangencial dos vasos; a influência da interação dos efeitos idade x posição radial, no sentido medula-casca, também foi verificada.

Conforme pode ser observado no Quadro 10, os valores encontrados neste trabalho estão em conformidade com os normalmente encontrados na literatura, mostrando um aumento no diâmetro tangencial dos vasos em função da variação da idade e da posição radial, no sentido medula-casca. O valor médio do diâmetro tangencial dos vasos foi de $122,2 \mu \mathrm{m}$, sendo os limites individuais superior e inferior, respectivamente, de 162,3 $\mu \mathrm{m}$ (idade de 20 anos, na posição mais próxima da casca) e 76,8 $\mu \mathrm{m}$ (idade de 10 anos na posição mais próxima da medula), com uma variação de $211,3 \%$. Estatisticamente, verificaram-se diferenças entre a madeira de 10, 20 e 25 anos, mas tais diferenças não foram observadas entre as madeiras de 10 e 14 anos, bem como entre as madeiras de $14 \mathrm{e} 25$ anos. Quanto à variação radial, não se observaram diferenças significativas entre as posições correspondentes a 75 e $100 \%$ no sentido medula-casca, mas tais diferenças foram observadas entre todas as demais posições.

Quadro 5 - Análise de variância do diâmetro do lume das fibras da madeira de Eucalyptus grandis de diferentes idades e posições, no sentido medula-casca

Table 5 - Variance analysis of the fiber lumen diameter of Eucalyptus grandis wood of different ages and distances in the pith-to-bark direction

\begin{tabular}{lcccc}
\hline Causa da variação & G. L. & Soma quadrados & Q. médio & F \\
\hline A: Idade & 3 & 6151,96 & 2050,65 & $182,88 *$ \\
B: Medula-casca & 4 & 1660,73 & 15,183 & $37,03 *$ \\
AB & 12 & 3774,15 & 314,513 & $28,05 *$ \\
Resíduo & 4778 & 53576,0 & 11,2131 & 0,0000 \\
Total (Corrigido) & 4797 & 65159,4 & & \\
\hline
\end{tabular}

C.V. experimental: $27,34 \%$. NOTA: $*$ significativo a de $95 \%$ de probabilidade.

R. Árvore, Viçosa-MG, v.31, n.6, p.1081-1090, 2007 
Influência da idade e da posição radial nas dimensões ...

Quadro 6 - Dimensões do diâmetro do lume das fibras (mm) da madeira de Eucalyptus grandis Hill ex. Maiden, de diferentes idades (anos) e posições no sentido medula-casca

Table 6 - Dimensions of lumen diameter ( $\mathrm{mm}$ ) of Eucalyptus grandis Hill ex. Maiden wood fiber of different ages (yrs) and distances in the pith-to-bark direction

\begin{tabular}{|c|c|c|c|c|c|}
\hline Tratamento & $\mathrm{N}$ & Médias & Tukey & $\mathrm{CV}(\%)$ & $\mathrm{DP}(\mu \mathrm{m})$ \\
\hline Idade 20 anos & 320 & 10,64 & $\mathrm{a}^{\frac{1}{\prime}}$ & 29,00 & 3,087 \\
\hline Idade 25 anos & 320 & 11,84 & $\mathrm{~b}$ & 27,27 & 3,228 \\
\hline Idade 10 anos & 320 & 12,85 & $\mathrm{c}$ & 30,29 & 3,891 \\
\hline Idade 14 anos & 320 & 13,67 & $\mathrm{c}$ & 27,51 & 3,761 \\
\hline Posição 0\% (M-C) & 400 & 11,79 & a & 23,92 & 2,821 \\
\hline Posição $25 \%$ (M-C) & 400 & 11,79 & a & 30,39 & 3,583 \\
\hline Posição 50\% (M-C) & 400 & 11,80 & a & 36,45 & 4,301 \\
\hline Posição $75 \%$ (M-C) & 400 & 12,63 & $\mathrm{~b}$ & 27,62 & 3,488 \\
\hline Posição $100 \%$ (M-C) & 400 & 13,23 & $\mathrm{c}$ & 29,07 & 3,847 \\
\hline \multicolumn{6}{|c|}{ Interação idade X posição radial medula-casca } \\
\hline 25 anos $\mathrm{x}$ posição $0 \%$ & 80 & 9,45 & $\mathrm{a}$ & 33,26 & 3,142 \\
\hline 20 anos $\times$ posição $0 \%$ & 80 & 9,62 & $\mathrm{a}$ & 35,77 & 3,440 \\
\hline 20 anos $\mathrm{x}$ posição $25 \%$ & 80 & 9,87 & $a b$ & 29,66 & 2,926 \\
\hline 20 anos x posição $50 \%$ & 80 & 10,85 & $\mathrm{bc}$ & 24,43 & 2,651 \\
\hline 20 anos $x$ posição $75 \%$ & 80 & 11,09 & $\mathrm{~cd}$ & 28,78 & 3,191 \\
\hline 14 anos $\mathrm{x}$ posição $0 \%$ & 80 & 11,45 & $\mathrm{~cd}$ & 34,12 & 3,908 \\
\hline 10 anos $\mathrm{x}$ posição $0 \%$ & 80 & 11,61 & $\mathrm{~cd}$ & 23,04 & 2,675 \\
\hline 25 anos x posição $25 \%$ & 80 & 11,70 & $\mathrm{~cd}$ & 22,06 & 2,581 \\
\hline 20 anos x posição $100 \%$ & 80 & 11,79 & $\mathrm{~cd}$ & 22,38 & 2,638 \\
\hline 25 anos x posição $50 \%$ & 80 & 12,01 & de & 25,93 & 3,114 \\
\hline 14 anos x posição $25 \%$ & 80 & 12,06 & de & 27,49 & 3,316 \\
\hline 25 anos $x$ posição $75 \%$ & 80 & 12,98 & ef & 21,82 & 2,834 \\
\hline 14 anos $x$ posição $50 \%$ & 80 & 13,04 & ef & 29,06 & 3,789 \\
\hline 25 anos x posição $100 \%$ & 80 & 13,05 & ef & 23,53 & 3,071 \\
\hline 14 anos x posição $75 \%$ & 80 & 13,51 & fg & 26,40 & 3,566 \\
\hline 10 anos x posição $25 \%$ & 80 & 13,64 & fgh & 28,29 & 3,859 \\
\hline 10 anos $\mathrm{x}$ posição $50 \%$ & 80 & 13,85 & fgh & 22,69 & 3,142 \\
\hline 14 anos x posição $100 \%$ & 80 & 14,16 & gh & 29,85 & 4,228 \\
\hline 10 anos x posição $75 \%$ & 80 & 14,63 & $\mathrm{~h}$ & 29,39 & 4,300 \\
\hline 10 anos x posição $100 \%$ & 80 & 14,63 & $\mathrm{~h}$ & 25,97 & 3,800 \\
\hline Média Geral & & & 12,25 & & \\
\hline
\end{tabular}

1/ Médias seguidas da mesma letra não diferem estatisticamente entre si, pelo teste de Tukey com significância de $5 \%$ ( $<<0,05$ ). $\mathrm{M}-\mathrm{C}=$ medula-casca; $\mathrm{CV}=$ coeficiente de variação $(\%) ;$ e DP = desvio-padrão.

Quadro 7 - Análise de variância da espessura da parede do diâmetro do lume das fibras da madeira de Eucalyptus grandis de diferentes idades e posições, no sentido medula-casca

Table 7 - Variance analysis of fiber lumen diameter wall thickness of Eucalyptus grandis wood ofdifferent ages and at different distances in the pith-to-bark direction

\begin{tabular}{lccccc}
\hline Causa da variação & G. L. & Soma quadrados & Q. médio & F & P \\
\hline A:Idade & 3 & 296,655 & 98,885 & $77,66^{*}$ & 0,0000 \\
B: Medula-casca & 4 & 1310,86 & 327,715 & $257,37^{*}$ & 0,0000 \\
AB & 12 & 35,244 & 28,7703 & $22,59^{*}$ & 0,0000 \\
Resíduo & 4778 & 6083,99 & 1,27333 & & \\
\hline Total (Corrigido) & 4797 & 8036,92 & & & \\
\hline
\end{tabular}

C.V. experimental: $30,01 \%$. NOTA: * significativo a $95 \%$ de probabilidade. 
Quadro 8-Espessura da parede das fibras ( $\mathrm{mm}$ ) da madeira de Eucalyptus grandis Hill ex. Maiden, de diferentes idades (anos) e posições no sentido medula-casca

Table 8 -Fiber wall thickness ( $\mathrm{mm}$ ) of Eucalyptus grandis Hill ex. Maiden wood of different ages (years) and at different distances in the pith-to-bark direction

\begin{tabular}{|c|c|c|c|c|c|}
\hline Tratamento & $\mathrm{N}$ (amostras) & Médias & Tukey & $\mathrm{CV}(\%)$ & $\mathrm{DP}(\mu \mathrm{m})$ \\
\hline Idade 10 anos & 320 & 3,37 & $a^{1^{\prime \prime}}$ & 31,59 & 1,063 \\
\hline Idade 14 anos & 320 & 3,78 & $\mathrm{~b}$ & 31,81 & 1,226 \\
\hline Idade 25 anos & 320 & 3,85 & $\mathrm{c}$ & 37,15 & 1,403 \\
\hline Idade 20 anos & 320 & 4,05 & $\mathrm{~d}$ & 33,65 & 1,362 \\
\hline Posição 0\% (M-C) & 400 & 3,16 & a & 30,33 & 0,958 \\
\hline Posição 25\% (M-C) & 400 & 3,34 & $\mathrm{~b}$ & 31,10 & 1,039 \\
\hline Posição 50\% (M-C) & 400 & 3,55 & $\mathrm{c}$ & 30,66 & 1,088 \\
\hline Posição 75\% (M-C) & 400 & 4,28 & d & 31,30 & 1,338 \\
\hline Posição 100\% (M-C) & 400 & 4,48 & $\mathrm{e}$ & 31,89 & 1,429 \\
\hline \multicolumn{6}{|c|}{ Interação idade $\mathrm{X}$ posição radial medula-casca } \\
\hline 10 anos $\mathrm{x}$ posição $0 \%$ & 80 & 3,01 & $\mathrm{a}$ & 24,82 & 0,748 \\
\hline 14 anos $x$ posição $0 \%$ & 80 & 3,03 & a & 29,36 & 0,889 \\
\hline 10 anos x posição $25 \%$ & 80 & 3,09 & a & 30,72 & 0,948 \\
\hline 25 anos $x$ posição $0 \%$ & 80 & 3,11 & $a b$ & 32,79 & 1,019 \\
\hline 25 anos x posição $25 \%$ & 80 & 3,22 & abc & 28,44 & 0,916 \\
\hline 10 anos x posição $50 \%$ & 80 & 3,27 & abcd & 33,57 & 1,096 \\
\hline 25 anos x posição $50 \%$ & 80 & 3,38 & abcd & 31,84 & 1,075 \\
\hline 20 anos x posição $0 \%$ & 80 & 3,46 & bcd & 30,48 & 1,054 \\
\hline 20 anos $x$ posição $25 \%$ & 80 & 3,48 & cde & 30,74 & 1,070 \\
\hline 10 anos $x$ posição $75 \%$ & 80 & 3,54 & cde & 28,99 & 1,027 \\
\hline 14 anos $x$ posição $25 \%$ & 80 & 3,55 & cde & 26,69 & 0,947 \\
\hline 14 anos x posição $50 \%$ & 80 & 3,60 & de & 31,95 & 1,151 \\
\hline 10 anos x posição $100 \%$ & 80 & 3,83 & ef & 27,09 & 1,038 \\
\hline 20 anos x posição $50 \%$ & 80 & 4,01 & $\mathrm{f}$ & 27,01 & 1,083 \\
\hline 14 anos $x$ posição $75 \%$ & 80 & 4,10 & $\mathrm{f}$ & 30,56 & 1,245 \\
\hline 25 anos $x$ posição $75 \%$ & 80 & 4,19 & fg & 30,90 & 1,293 \\
\hline 14 anos x posição $100 \%$ & 80 & 4,53 & gh & 29,60 & 1,341 \\
\hline 20 anos x posição $75 \%$ & 80 & 4,85 & hi & 27,05 & 1,311 \\
\hline 25 anos x posição $100 \%$ & 80 & 4,92 & $\mathrm{i}$ & 28,21 & 1,387 \\
\hline 20 anos x posição $100 \%$ & 80 & 5,07 & $\mathrm{i}$ & 31,37 & 1,591 \\
\hline
\end{tabular}

1/ Médias seguidas da mesma letra não diferem estatisticamente entre si, pelo teste de Tukey com significância de $5 \%$ ( $<<0,05$ ). $\mathrm{M}-\mathrm{C}=$ medula-casca; $\mathrm{CV}=$ coeficiente de variação $(\%)$; e DP = desvio padrão.

Quadro 9 - Análise de variância do diâmetro tangencial dos vasos da madeira de Eucalyptus grandis de diferentes idades e posições, no sentido medula-casca

Table 9 - Variance analysis of tangential diameter vessels of Eucalyptus grandis wood of different ages and at different distances in the pith-to-bark direction

\begin{tabular}{lccccc}
\hline Causa da variação & G. L. & Soma quadrados & Q. médio & F & P \\
\hline A: Idade & 3 & 16395,45 & 5465,15 & $22,517^{*}$ & 0,0000 \\
B: Medula-Casca & 4 & 113042,6 & 28260,65 & $116,439^{*}$ & 0,0000 \\
AB & 12 & 7439,773 & 249,2007 & $14,25^{*}$ & 0,0000 \\
Resíduo & 232 & 56308,06 & 242,7072 & & \\
\hline Total (Corrigido) & 239 & 185746,1 & & & \\
\hline
\end{tabular}

C.V. experimental: $12,75 \%$. NOTA: * significativo a $95 \%$ de probabilidade.

R. Árvore, Viçosa-MG, v.31, n.6, p.1081-1090, 2007 
Quadro 10 -Diâmetro tangencial dos vasos (mm) da madeira Eucalyptus grandis Hill ex. Maiden de diferentes idades (anos) e posições, no sentido medula-casca

Table 10 - Vessel tangential diameter $(\mathrm{mm})$ of Eucalyptus grandis Hill ex. Maiden wood of different ages (years) and at different distances in the pith-to-bark direction

\begin{tabular}{|c|c|c|c|c|c|}
\hline Tratamento & $\mathrm{N}$ (amostras) & Médias & Tukey & $\mathrm{CV}(\%)$ & $\mathrm{DP}(\mu \mathrm{m})$ \\
\hline Idade 10 anos & 64 & 113,3 & $\mathrm{a}^{\mathbf{1}^{\prime}}$ & 24,06 & 27,305 \\
\hline Idade 14 anos & 64 & 118,5 & $a b$ & 21,48 & 25,449 \\
\hline $\begin{array}{ll}\text { Idade } & 25 \text { anos } \\
\text { Idade } & 20 \text { anos }\end{array}$ & $\begin{array}{l}64 \\
64\end{array}$ & $\begin{array}{l}121,5 \\
135,6\end{array}$ & $\begin{array}{l}\mathrm{b} \\
\mathrm{c}\end{array}$ & $\begin{array}{l}19,68 \\
22,19\end{array}$ & $\begin{array}{l}23,915 \\
30,086\end{array}$ \\
\hline Posição $0 \%$ (M-C) & 80 & 87,1 & a & 20,15 & 17,552 \\
\hline Posição 25\% (M-C) & 80 & 109,4 & $\mathrm{~b}$ & 13,97 & 15,283 \\
\hline Posição 50\% (M-C) & 80 & 127,6 & $\mathrm{c}$ & 12,14 & 15,496 \\
\hline Posição 75\% (M-C) & 80 & 139,5 & d & 13,67 & 19,065 \\
\hline Posição $100 \%$ (M-C) & 80 & 147,4 & $\mathrm{~d}$ & 13,60 & 20,041 \\
\hline \multicolumn{6}{|c|}{ Interação idade $X$ posição radial medula-casca } \\
\hline 10 anos $\mathrm{x}$ posição $0 \%$ & 16 & 77,9 & $\mathrm{a}$ & 16,22 & 12,637 \\
\hline 14 anos $\mathrm{x}$ posição $0 \%$ & 16 & 88,4 & a & 25,97 & 22,958 \\
\hline 25 anos $\mathrm{x}$ posição $0 \%$ & 16 & 88,8 & a & 17,27 & 15,340 \\
\hline 20 anos $\mathrm{x}$ posição $0 \%$ & 16 & 93,5 & $\mathrm{a}$ & 17,00 & 15,895 \\
\hline 10 anos $x$ posição $25 \%$ & 16 & 98,2 & $a b$ & 8,11 & 7,965 \\
\hline 14 anos x posição $25 \%$ & 16 & 107,6 & $\mathrm{~b}$ & 17,41 & 18,736 \\
\hline 25 anos x posição $25 \%$ & 16 & 110,1 & $\mathrm{~b}$ & 9,27 & 10,211 \\
\hline 20 anos x posição $25 \%$ & 16 & 121,7 & c & 10,77 & 13,110 \\
\hline 25 anos $x$ posição $50 \%$ & 16 & 122,1 & $\mathrm{c}$ & 7,66 & 9,355 \\
\hline 10 anos $x$ posição $50 \%$ & 16 & 122,5 & $\mathrm{c}$ & 10,36 & 12,688 \\
\hline 14 anos $x$ posição $50 \%$ & 16 & 122,8 & c & 12,18 & 14,962 \\
\hline 10 anos $x$ posição $75 \%$ & 16 & 131,2 & $\mathrm{~d}$ & 14,33 & 18,801 \\
\hline 14 anos x posição $75 \%$ & 16 & 131,5 & $\mathrm{~d}$ & 11,97 & 15,745 \\
\hline 10 anos x posição $100 \%$ & 16 & 136,6 & de & 18,13 & 24,767 \\
\hline 25 anos x posição $75 \%$ & 16 & 137,7 & de & 8,55 & 11,774 \\
\hline 14 anos x posição $100 \%$ & 16 & 142,0 & $\mathrm{e}$ & 9,89 & 14,038 \\
\hline 20 anos x posição $50 \%$ & 16 & 143,1 & $\mathrm{e}$ & 9,99 & 14,296 \\
\hline 25 anos x posição $100 \%$ & 16 & 148,9 & $\mathrm{f}$ & 6,24 & 9,293 \\
\hline 20 anos x posição $75 \%$ & 16 & 157,6 & $\mathrm{~g}$ & 11,11 & 17,503 \\
\hline 20 anos x posição $100 \%$ & 16 & 161,9 & $\mathrm{~g}$ & 12,76 & 20,656 \\
\hline
\end{tabular}

I/ Médias seguidas da mesma letra não diferem estatisticamente entre si, pelo teste de Tukey com significância de 5\% (p<0,05). ). M$\mathrm{C}=$ medula-casca; $\mathrm{CV}=$ coeficiente de variação $(\%) ;$ e DP = desvio-padrão.

\section{CONCLUSÕES}

Com base nos resultados, pode-se concluir que:

a) As dimensões das fibras e dos vasos apresentaram variações tanto em relação à idade quanto ao sentido medula-casca.

b) As variações observadas na posição radial sentido medula-casca foram mais destacadas que as observadas entre as diferentes idades.

c) O comprimento, a espessura da parede das fibras e o diâmetro dos vasos mostraram-se positivamente correlacionados com a idade e com a posição radial no sentido medula-casca. d) A largura e o diâmetro do lume das fibras correlacionaram-se negativamente com a idade, mas positivamente com a posição radial, no sentido medula-casca.

\section{5 - REFERÊNCIAS}

ANDRADE, M. C. D. Variação das características das fibras em função da densidade básica de cavacos de madeira de Eucalyptus grandis Hill ex.Maiden. Botucatu(SP).1987. 43f. Dissertação (Mestrado em Ciência Florestal) - Universidade Estadual de São Paulo, Botucatu, 1987.

R. Árvore, Viçosa-MG, v.31, n.6, p.1081-1090, 2007 
DADSWELL, H. E. The anatomy of eucalypt wood. Melbourne: Forest Products Laboratory, CSIRO, n.66, p.1-28, 1972.

ESTEBAn, L. G. et al. Anatomia y su identificación de espécies a nível de espécie. Madri: AMVE Ediciones, 2002. 421 p.

ESTEBAN, L. G. et al. Madera y su anatomia. Madri: AMVE Ediciones, 2003. 327p.

\section{FOREST PRODUCTS LABORATORY. Wood}

Handbook - wood as an engineering material. Madison: US Department of Agriculture, Forest Service, 1999. 463p.

MARCATI, C. R. Estudo da anatomia e das propriedades tecnológicas da madeira do angico-vermelho (Piptadenia peregrina Benth). 1992. 94f. Dissertação (Mestrado em Ciências Florestais) - Universidade Federal de Viçosa, Viçosa, MG, 1992.

MOREIRA, W. S. Relações entre propriedades físico-mecânicas e características anatômicas e químicas da madeira. 1999. 107f. Tese (Doutorado em Ciências Florestais) - Universidade Federal de Viçosa, Viçosa, MG, 1999.

OLIVEIRA, J. T. S. Caracterização da madeira de eucalipto para a construção civil. 1997. 2.v. 429f. Tese (Doutorado em Engenharia Civil) - Escola Politécnica, Universidade de São Paulo, São Paulo, 1997.
SHIMOYAMA, V. R. S. Variações da densidade básica e características anatômicas e químicas da madeira em Eucalyptus sp. 1990. 93f. Dissertação (Mestrado em Engenharia Florestal) - Escola Superior de Agricultura “Luiz de Queiroz”, Piracicaba, 1990.

SILVA, J. C. Caracterização da Madeira de Eucalyptus grandis Hill ex Maiden, de diferentes idades, visando a sua utilização na indústria moveleira. 2002. 160f. Tese (Doutorado em Engenharia Florestal) Universidade Federal do Paraná, Curitiba, 2002.

SILVA, J. R. M.; LIMA, J. T.; TRUGILHO, P. F. Usinabilidade da madeira de Eucalyptus grandis em diferentes regiões da medula à casca. Cerne, v.13, n.1, p.25-31, 2007.

TOMAZELLO FILHO, M. Estrutura anatômica da madeira de oito espécies de eucalipto cultivadas no Brasil. IPEF, v.29, p.25-36, 1985a.

TOMAZELLO FILHO, M. Variação radial da densidade básica e da estrutura anatômica da madeira de Eucalyptus saligna e Eucalyptus grandis. IPEF, v.29, p.37-45, 1985b.

TSOUMIS, G. Science and technology of wood: Structure, properties and utilization. New York: Van Nostrand Reinold, 1991. 494p.

WILKES, J. Variations in wood anatomy within of Eucalyptus. IAWA Bulletin, v.9, n.1, p.13-23, 1988.

ZOBEL, J. B.; BUJTENEN, J. P. Wood

variation: Its causes and control. New York: Springer-Verlag, 1989.363p. 